\title{
Anisotropic Multipolar Exchange Interactions in Systems with Strong Spin-Orbit Coupling
}

\author{
Shu-Ting Pi, ${ }^{1, \oplus}$ Ravindra Nanguneri, ${ }^{1,2, \text {, }}$ and Sergey Savrasov ${ }^{1, \text {, }}$ \\ ${ }^{1}$ Department of Physics, University of California, Davis, \\ One Shields Avenus, Davis, California 95616 USA \\ ${ }^{2}$ Department of Chemistry and Biochemistry, University of Notre Dame, Notre Dame, Indiana, 46556 USA
}

(Dated: May 25, 2022)

\begin{abstract}
We introduce a theoretical framework for computaions of anisotropic multipolar exchange interactions found in many spin-orbit coupled magnetic systems and propose a method to extract these coupling constants using a density functional total energy calculation. This method is developed using a multipolar expansion of local density matrices for correlated orbitals that are responsible for magnetic degrees of freedom. Within the mean-field approximation, we show that each coupling constant can be recovered from a series of total energy calculations via what we call the "pair-flip" technique. This technique flips the relative phase of a pair of multipoles and computes corresponding total energy cost associated with the given exchange constant. To test it, we apply our method to Uranium Dioxide, which is a system known to have pseudospin $J=1$ superexchange induced dipolar, and superexchange plus spin-lattice induced quadrupolar orderings. Our calculation reveals that the superexchange and spin-lattice contributions to the quadrupolar exchange interactions are about the same order with ferro- and antiferro-magnetic contributions, respectively. This highlights a competition rather than a cooperation between them. Our method could be a promising tool to explore magnetic properties of rare-earth compounds and hidden-order materials.
\end{abstract}

\section{INTRODUCTION}

Solid-state systems with strong spin-orbit coupling have been a research frontier for decades due to their rich magnetic phases that cannot be explained by simplified model Hamiltonians. Among their peculiar properties, the existence of multipolar moments may be one characteristics most inaccessible to experimental investigation ${ }^{\underline{1}}$. Interactions between such moments not only induce complexity in high-rank magnetic order as observed in $\mathrm{LaFeAsO}_{2,3}^{2,3}, \mathrm{PrFe}_{4} \mathrm{P}_{12} \underline{\underline{4}, 5}, \mathrm{UPt}_{3}{ }_{3}, \mathrm{YbRu}_{2} \mathrm{Ge}_{2}{ }^{7}$, $\mathrm{UO}_{2} \underline{10}-13,44$ and many other compounds $\stackrel{89}{ }$ but also exhibit the phenomena of hidden order phases as observed in $\mathrm{NpO}_{2} \stackrel{14-16}{=}, \mathrm{Ce}_{1-x} \mathrm{La}_{x} \mathrm{~B}_{6} \frac{17}{2}$ and $\mathrm{URu}_{2} \mathrm{Si}_{2} \underline{\underline{18}} \underset{22}{2}$. Because of the active orbital degrees of freedom, the conventional $S=1 / 2$ Heisenberg model ${ }^{23}$ is no longer adequate to describe their magnetic moments and, instead, highrank tensor operators are required to form a complete basis ${ }^{1}$. The introduction of multipolar moments makes the exchange interactions complicated, with a great number of coupling constants, and makes their computation a difficult problem in condensed matter physics.

Earlier studies of the exchange interactions in spinorbital systems have been developed by Coqblin and Schrieffer. They implemented the Schrieffer-Wolff transformation on a spin-orbit coupled Anderson lattice model and transformed it to a Kondo lattice problem so that the RKKY interaction could be deduced $24-28$. Unlike conventional $S=1 / 2$ Heisenberg model where the RKKY interaction is isotropic $\underline{41}$, the RKKY interaction for the spin-orbital model has an intrinsic anisotropy even in a homogeneous system. In the 80's, Cooper et. al. solved the Coqblin-Schrieffer Hamiltonian for $4 f^{1}$ Cerium monopnictides and explained their many unusual properties that conventional exchange models failed to reproduce $\underline{29}-34$. In the 90 's, they further suggested a first-principles scheme to calculate the coupling constants of a few simple materials and obtained satisfactory results $35-39$. Although we now have a better understanding about the multipolar exchange interactions nowadays, a comprehensive physical picture remains lacking. Most of the models and computational schemes are either based on the knowledge of specific exchange mechanisms or too complicated to apply for materials. In this paper, we propose a method to compute the multipolar coupling constants using a total energy electronic structure calculation based on density functional theory (DFT) in its local density approximation (LDA) or using an LDA+U approach $\frac{42}{2}$. A short account of the present work has appeared already $\stackrel{44}{\underline{4}}$.

We begin with a quick review of the RKKY interaction in spin-orbital systems in Sec.II. These works were mostly contributed by Coqblin, Schrieffer and Cooper and we emphasize the mechanism that induces the intrinsic anisotropy of the exchange interactions. The formulation of multipolar tensor operators is given in Sec.III. The language of multipolar tensor operators is the most natural one to describe spin-orbit coupled systems. Using this language, density matrices can be split into scalar, dipolar, quadrupolar and higher multipolar components based on their rotational symmetry. The complicated exchange coupling matrix may become simplified and even diagonal when expressed in this tensor space. In Sec.IV, an efficient method to deduce coupling constants using the $\mathrm{LDA}+\mathrm{U}$ electronic-structure calculation is introduced. We call this method the "pair-flip technique" because it relates every coupling parameter to a series of total energy calculations by flipping the relative phase of a multipole pair. Application to Uranium Dioxide $\left(\mathrm{UO}_{2}\right)$, is discussed in Sec.V. $U O_{2}$ being famous for its important 


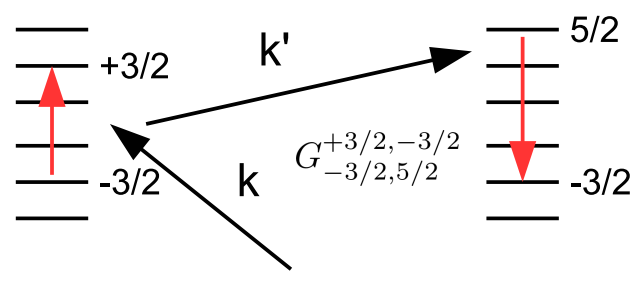

FIG. 1. (color) The RKKY mechanism of a $J=5 / 2$ system: an incoming free electron with crystal momentum $k$ interacts with a local moment and induces a transition between the degenerate states $-3 / 2$ to $3 / 2$. Then it leaves with $k^{\prime}$ and induces another transition $5 / 2$ to $-3 / 2$ at a neighboring site. These transitions are coupled by the exchange constant $G_{-3 / 2,5 / 2}^{+3 / 2,-3 / 2}$

applications in nuclear energy industry, is known to have pseudospin $J=1$ ground state, with both dipolar and quadrupolar moment orderings, and therefore is a good candidate to test our method. We find the superexchange contribution in $\mathrm{UO}_{2}$ tends to be ferromagnetic, which is very different from past studies. We conclude in Sec.VI by speculating that our method could be a promising tool to explore other spin-orbit coupled systems and materials with the hidden order.

\section{SPIN-ORBIT COUPLED EXCHANGE INTERACTIONS}

\section{A. Exchange Interactions}

Exchange interaction appears in an effective model for an Anderson lattice Hamiltonian in its low excitation limit where the particle fluctuation is frozen and only transitions among the internal degrees of freedom, i.e. the degenerate single particle states, are allowed. The Anderson lattice model ${ }^{40.43}$ is given by

$$
\begin{aligned}
H & =\sum_{\mathbf{k} \sigma} \epsilon_{\mathbf{k} \sigma}+\sum_{d}\left\{\epsilon_{d} n_{d \sigma}+U n_{d \uparrow} n_{d \downarrow}\right\} \\
& +\sum_{\mathbf{k} d \sigma}\left\{V_{\mathbf{k} d} c_{\mathbf{k} \sigma}^{\dagger} c_{d \sigma}+h . c\right\}
\end{aligned}
$$

where $d$ is the localized correlated state, $\epsilon_{d}$ is the on-site energy of the localized $d$ orbital, $\mathbf{k}$ is the crystal momentum, $\sigma$ is the spin index, $U$ is the Hubbard interaction, $V$ is the coupling between a conduction electron and a localized $d$ state. Let us denote the first two terms as $H_{0}$ and the last one as $H_{1}$. In the Kondo limit $U \gg \epsilon_{d}$, charge transfer is frozen and the Anderson lattice model becomes the Kondo lattice model. In the 60's, Schrieffer and Wolff suggested a procedure to eliminate the charge fluctuation effects 24 (high order perturbation of $H_{1}$ ) by introducing a unitary transformation that keeps $H$ to $\mathcal{O}\left(H_{1}\right)$ only, $H^{\prime}=e^{s} H e^{-s} \sim \mathcal{O}\left(H_{1}\right)$. It requires

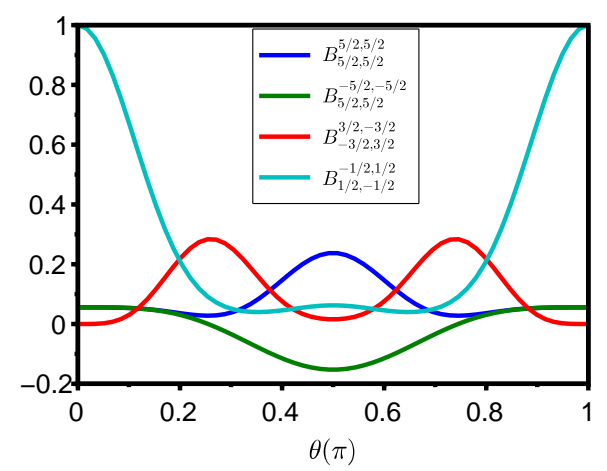

FIG. 2. (color) The angular dependence of the coupling constants $B_{\gamma \alpha}^{\delta \beta}(\theta, \phi)$ as a function of $\theta$ (in unit of $\pi$ ) with $\phi=0$. Anisotropy can be found in all transitions.

$\left[H_{0}, s\right]=H_{1}$ and $H^{\prime}=H_{0}+\frac{1}{2}\left[s, H_{1}\right]$. Coqblin and Schrieffer implemented this transformation to a spinorbit coupled $J=5 / 2$ Cerium $\left(4 f^{1}\right)$ system and derived the spin-orbital version of RKKY interaction which describes the exchange interaction between the two local moments 25,26 .

A general form of the two-ion exchange interaction in a spin-orbit coupled lattice system can be written as 1

$$
H(J)=\sum_{i j} \sum_{\alpha \beta \gamma \delta} G_{\gamma \alpha}^{\delta \beta}(i, j) c_{i \delta}^{\dagger} c_{i \gamma} c_{j \beta}^{\dagger} c_{j \alpha}
$$

where $i, j$ are site indices, $\alpha, \beta, \gamma, \delta$ are labels of the degenerate states which range from $-J$ to $J, J$ being the quantum number of the total moment. The physics of this Hamiltonian is easy to understand (see Fig.1). It describes the transition from $\alpha$ to $\beta$ at site $i$ and another transition from $\gamma$ to $\delta$ at site $j$. These transitions are coupled by a constant $G_{\gamma \alpha}^{\delta \beta}(i, j)$. There are many mechanisms to induce these transitions, e.g. RKKY (interaction with conduction electrons), superexchange (interaction with neighboring non-magnetic atoms), and spin-lattice coupling (interaction with lattice vibrations $)^{41}$.

\section{B. Anisotropy}

A major feature of the spin-orbit coupled exchange interaction is its anisotropy. To show this, let us consider a simple but still realistic model (e.g. Cerium compounds) that each site has $f^{1}$ configuration with $J=5 / 2$ ground state. As given by Ref $\stackrel{30}{ }$, the coupling matrix induced by the RKKY mechanism has the following form:

$$
H=\sum_{i j} E\left(\left|r_{i j}\right|\right) \sum_{\alpha \beta \gamma \delta} B_{\gamma \alpha}^{\delta \beta}(\theta, \phi) L_{\delta \gamma}^{i} L_{\beta \alpha}^{i}
$$




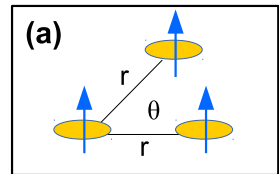

(d)

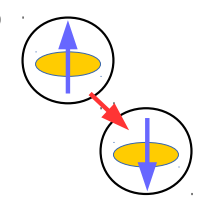

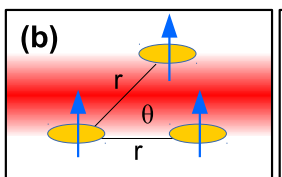

(e)

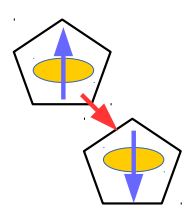

(c)

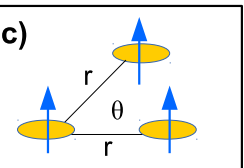

(f)

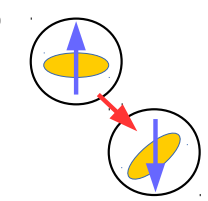

FIG. 3. (color) Anisotropy of exchange interactions. The blue arrows are local moments and the yellow ellipses are orbitals. (a) $\mathrm{S}=1 / 2$ in a homogeneous background (b) $\mathrm{S}=1 / 2$ in an inhomogeneous background. The anisotropy can be induced by many factors, e.g. crystal structure, electronic structure and external fields. (c) $\mathrm{J}=5 / 2$ in a homogeneous background. In (d)-(f), we use a cage around the moment to represent the relation between the background and the local moment as described by (a)-(c) respectively: (d) Transition of the spin moment in a homogeneous background will not change anything; the exchange interaction is isotropic, (e) Transition of the moment in an inhomogeneous background gives a different configuration; the exchange interaction is anisotropic (f) Transition of a spin-orbit coupled moment in a homogeneous background: Although the background has no directional dependence, due to highly anisotropic shapes of the orbitals exchange interaction becomes directionally dependent.

with

$$
\begin{aligned}
& \sum_{\gamma \alpha}^{\delta \beta}(\theta, \phi)=e^{i(\delta-\gamma+\beta-\alpha) \phi} \times \\
& \sum_{M M^{\prime}= \pm 1 / 2}\left\{d_{\delta M^{\prime}}(\theta) d_{\gamma M}(\theta)-\frac{1}{6} \delta_{M M^{\prime}} \delta_{\delta \gamma}\right\} \\
& \times\left\{d_{\beta M}(\theta) d_{\alpha M^{\prime}}(\theta)-\frac{1}{6} \delta_{M M^{\prime}} \delta_{\beta \alpha}\right\}
\end{aligned}
$$

where $L_{\delta \gamma}^{i}=\left|\delta^{i}\right\rangle\left\langle\gamma^{i}\right|$ is the transition operator which is the single particle version of $c_{i \delta}^{\dagger} c_{i \gamma}, d_{\beta M}(\theta)$ is the quantum mechanical rotation matrix of $J=5 / 2$. Some matrix elements of the function $B_{\gamma \alpha}^{\delta \beta}(\theta, \phi)$ as a function of angle are shown in Fig.2.

Unlike conventional $S=1 / 2$ RKKY where the coupling matrix has only $E\left(\left|r_{i j}\right|\right)$ dependence ${ }^{41}$, one can immediately find that all the matrix elements are highly anisotropic for the spin-orbit coupled systems. For example, transitions $\left[\frac{1}{2} \rightarrow-\frac{1}{2}\right]$ and $\left[-\frac{1}{2} \rightarrow \frac{1}{2}\right]$ are strongly coupled only when two ions have relative angle $\theta=0$ or $\pi$ and become almost decoupled when $\theta=\pi / 4 \sim \pi / 3$. The physical origin of the intrinsic anisotropy comes from the spatial dependence of atomic orbitals. In Fig.3, we consider an exchange problem in a homogeneous and in an inhomogeneous systems. In (a) and (d), since the background (for RKKY, the background is the sea of conduction electrons) and the transition (varying the spin) are both homogeneous, so the exchange interaction is isotropic. In (b) and (e), the background is inhomogeneous, so a homogeneous transition still feels its envi-

ronmental anisotropy. In (c) and (f), even though the system is homogeneous, the transition (e.g. $-3 / 2$ to $1 / 2$ ) itself is always anisotropic due to its coupling with the spatial wave function (recall that $f$-orbitals have highly anisotropic shapes). The anisotropy induced by the active orbital degrees of freedom distinguishes the nature between a spin-only and a spin-orbit coupled exchange interaction and makes the calculation of the exchange matrix difficult due to the presence of many off-diagonal matrix elements.

\section{MULTIPOLAR TENSOR OPERATORS}

In the following, we use a single-particle description while extension to a many-body version can be achieved by replacing the ket and bra vectors by creation and annihilation operators.

\section{A. Super Basis}

An unit transition tensor operator in the total moment $J$ Hilbert space is defined as:

$$
L_{\delta \gamma}(J)=|\delta\rangle\langle\gamma|,
$$

where $\delta, \gamma$ are the magnetic moment states which range from $-J$ to $+J$. A matrix defined in the same Hilbert can be expressed in terms of the above operators, e.g., for $J=1 / 2$ we deal with $2 \times 2$ matrices and their expansions in terms of $L_{\delta \gamma}$ :

$$
\left(\begin{array}{ll}
1 & 2 \\
3 & 4
\end{array}\right)=1\left(\begin{array}{ll}
1 & 0 \\
0 & 0
\end{array}\right)+2\left(\begin{array}{ll}
0 & 1 \\
0 & 0
\end{array}\right)+3\left(\begin{array}{ll}
0 & 0 \\
1 & 0
\end{array}\right)+4\left(\begin{array}{ll}
0 & 0 \\
0 & 1
\end{array}\right) .
$$

Using the language above, we have:

$$
A=1 L_{11}+2 L_{12}+3 L_{21}+4 L_{22},
$$

where the coefficients can be obtained by taking the trace of the matrix and the daggered tensor operator : $\operatorname{Tr}\left[A L_{i j}^{\dagger}\right]$. This shows that $\left\{L_{\delta \gamma}(J)\right\}$ forms a basis with trace inner product and any operator defined in the same Hilbert space can be expanded in terms of this basis. In the following, we name the basis set that expands an operator defined in the $J$ Hilbert space with trace inner product as "super basis" to distinguish from the commonly used vector basis $\{|\delta\rangle\}$. The transition super basis is not only the option. A spherical harmonics super basis can be generated by ${ }^{1}$

$$
\begin{aligned}
Y_{K}^{Q}(J) & =\sum_{M M^{\prime}}(-1)^{J-M}(2 K+1)^{2} \\
& \times\left(\begin{array}{ccc}
J & J & K^{\prime} \\
M^{\prime} & M & Q
\end{array}\right)|J M\rangle\left\langle J M^{\prime}\right|,
\end{aligned}
$$

where the parentheses denote a $3 j$-symbol; $K$ is the rank which ranges $0 \sim 2 J ; Q$ is the projection index 
of rank $K$ which ranges from $-J$ to $+J$. Similarly a matrix defined in the $J$ Hilbert space has the property $A(J)=\sum_{K Q} \alpha_{K}^{Q} Y_{K}^{Q}(J)$ and the expansion coefficients can be calculated $\alpha_{K}^{Q}=\operatorname{Tr}\left[A Y_{K}^{\dagger Q}\right]$. One can easily verify that there are $(2 J+1)^{2}$ members in the spherical harmonics super basis which is exactly the number of matrix elements (also the number of members in the transition super basis) in the $J$ Hilbert space.

For the same example of $J=1 / 2$ we have

$$
\begin{aligned}
\left(\begin{array}{ll}
1 & 2 \\
3 & 4
\end{array}\right) & =\frac{5}{2}\left(\begin{array}{ll}
1 & 0 \\
0 & 1
\end{array}\right)+2\left(\begin{array}{ll}
0 & 1 \\
0 & 0
\end{array}\right) \\
& +\frac{3}{2}\left(\begin{array}{cc}
-1 & 0 \\
0 & 1
\end{array}\right)-3\left(\begin{array}{cc}
0 & 0 \\
-1 & 0
\end{array}\right) .
\end{aligned}
$$

Here, the spherical harmonics super basis is actually the unit, the $z$-projection, and the ladder (raising and lowering) operators with appropriate normalization constants: $Y_{0}^{0} \sim I, Y_{1}^{0} \sim \sigma^{z}, Y_{1}^{+1} \sim \sigma^{x}+i \sigma^{y}, Y_{1}^{-1} \sim \sigma^{x}-i \sigma^{y}$. It is called the spherical harmonics super basis because its members follow exactly the same symmetry as the spherical harmonics. $Y_{0}^{0}$ behaves like a $s$-orbital; $Y_{1}^{-1}, Y_{1}^{0}$ and $Y_{0}^{+1}$ behave like $p^{-1}, p^{0}$ and $p^{+1}$ orbitals.

In group theory, these operators are named after their rank: $K=0$ scalar, $K=1$ dipole, $K=2$ quadrupole, $K=3$ octupole, etc. We have to emphasize that the multipoles in this framework are different form those in the theory of electromagnetism, where the multipoles refer to the spatial distribution of charge $\rho(\mathbf{r})$ or magnetization $\mathbf{m}(\mathbf{r})$ expanded by multipolar functions $Y_{l}^{m}(\theta, \phi)$. Here, the multipoles do not refer to any spatial distribution but to the rotational properties of a matrix, or more precisely, to the transitions of magnetic moments. Although they follow the same algebra, they do not correspond to the same physical meaning.

Similarly, we can also define the cubic harmonics super basis, where all the operators are Hermitian 1

$$
\begin{aligned}
T_{K}^{Q} & =\frac{1}{\sqrt{2}}\left[(-1)^{Q} Y_{K}^{Q}(J)+Y_{K}^{-Q}(J)\right] \\
T_{K}^{-Q} & =\frac{i}{\sqrt{2}}\left[Y_{K}^{-Q}(J)-(-1)^{Q} Y_{K}^{-Q}(J)\right] .
\end{aligned}
$$

For $J=1 / 2$ case, these are Pauli matrices: $T_{0}^{0} \sim I$, $T_{1}^{+1} \sim \sigma_{y}, T_{1}^{0} \sim \sigma_{z}$ and $T_{0}^{0} \sim \sigma_{x}$. Also, this basis follows the same symmetry as cubic harmonics: $s, p^{x}, p^{y}$ and $p^{z}$. Therefore, instead of using abstract $(K, Q)$ indexes, we will label these tensor operators using their symmetry: $T^{s}, T^{x}, T^{y}, T^{z}, T^{x y}, T^{y z}, T^{z x}$, etc. It is straightforward to rewrite the spin-orbit coupled exchange interaction by using different super bases:

$$
\begin{aligned}
H & =\sum_{i j} \sum_{\alpha \beta \gamma \delta} G_{\gamma \alpha}^{\delta \beta}(i, j) L_{\delta \gamma}^{i}(J) L_{\beta \alpha}^{i}(J) \\
& =\sum_{i j} \sum_{K Q} F_{K_{i} K_{j}}^{Q i Q_{j}} Y_{Q_{i}}^{K_{i}}(J) Y_{Q_{j}}^{K_{j}}(J) \\
& =\sum_{i j} \sum_{K Q} C_{K_{i} K_{j}}^{Q i Q_{j}} T_{Q_{i}}^{K_{i}}(J) T_{Q_{j}}^{K_{j}}(J) .
\end{aligned}
$$

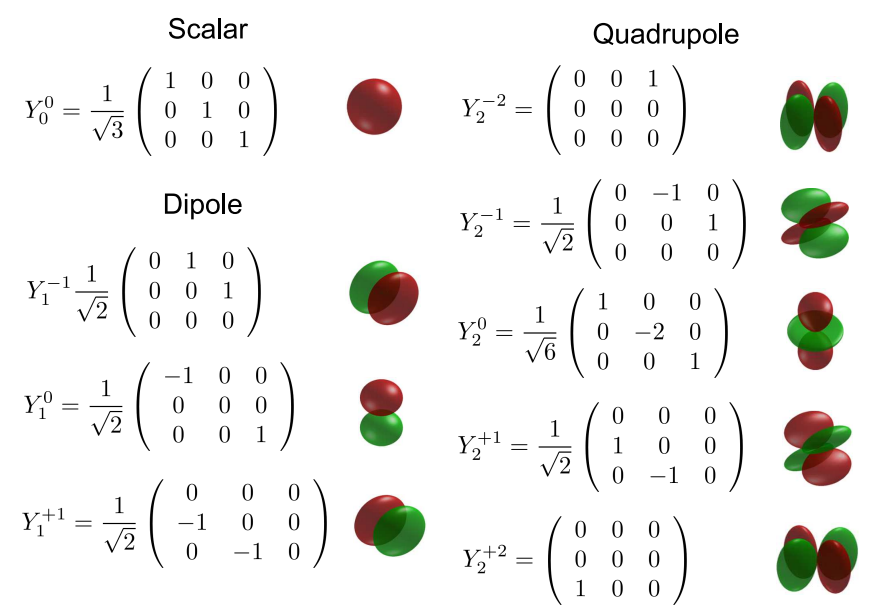

FIG. 4. (color) The spherical tensor operators of $J=1$ and the real part of analogous spherical harmonics. States to expand the matrices are ordered by $|-1\rangle,|0\rangle$ and $|+1\rangle$. Each member has its analogous spatial function as $s, p^{-1}, p^{0}, p^{+1}$, $d^{-2}, d^{-1}, d^{0}, d^{+1}$ and $d^{+2}$ spherical harmonics which follow the same symmetry under rotation.

The couplings among multipolar operators now appear naturally, and the coupling matrices in different bases can be linked by using unitary transformations. Obviously, one can define other super bases by making different linear combinations of them. The benefit of using appropriate super basis is that the coupling matrix may become block diagonal or even completely diagonal when using appropriate symmetry.

\section{B. Physical Pictures}

To illustrate the physics of multipolar tensor operators, let us focus on the spherical harmonics operators for $J=$ 1. In this case, we have 9 linearly independent tensor operators as shown in Fig.4. We also display the shapes of $s, p$ and $d$ spherical harmonics functions to represent their analogy. Because the rotational properties of those tensor operators are the same as the original spherical harmonics, we can "visualize" these matrices by this way.

Let us discuss the scalar term first. The scalar term is exactly an identity matrix, and since the identity is invariant under rotations, it always looks the same under any rotation as the $s$-orbital. An important feature of the scalar term is its relation to the total charge. If we expand the density matrix $\rho=\sum_{K, Q} \alpha_{K}^{Q} Y_{K}^{Q}$, the total charge of the system is proportional to the coefficient $\alpha_{0}^{0}$. As for the dipole terms, the matrices are no longer unchanged under rotation: $Y_{1}^{-1}$ and $Y_{1}^{+1}$ describe time-reversed transition processes which change a single quantum of the angular momentum. $Y_{1}^{0}$ is another diagonal matrix which induces no change of moment. Similar descriptions also hold for quadrupoles: $Y_{2}^{-2}$ and $Y_{2}^{+2}$ 


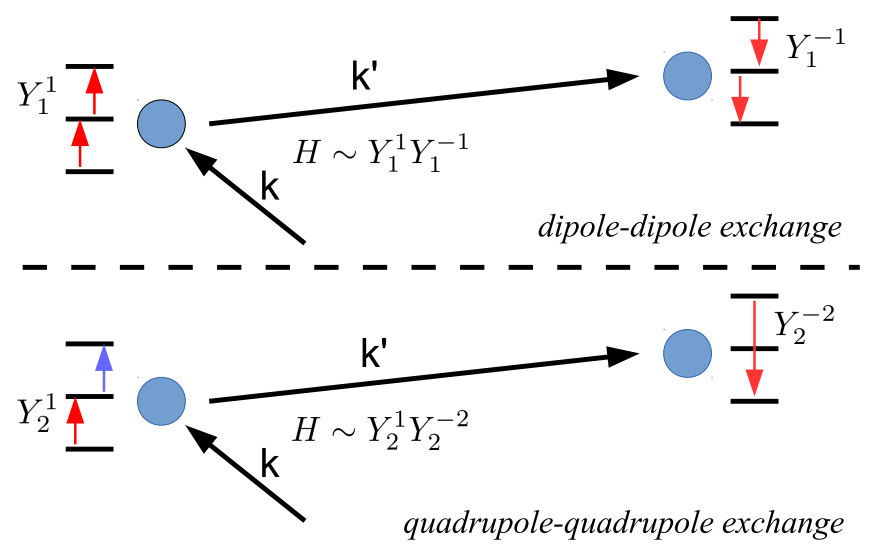

FIG. 5. (color) Dipole-dipole and quadrupole-quadrupole exchange interactions. An incoming conduction electron with momentrum $k$ interacts with a $J=1$ local moment, induces a $Y_{1}^{1}\left(Y_{2}^{1}\right)$ transition and leaves with momentum $k^{\prime}$ inducing a $Y_{1}^{-1}\left(Y_{2}^{-2}\right)$ transition on a neighboring site. The blue transition arrow for $Y_{2}^{1}$ means a negative phase compared to the red one.

change two moment quanta; $Y_{2}^{-1}$ and $Y_{2}^{+1}$ change one moment quantum; $Y_{2}^{0}$ changes no moment. Although $Y_{1}^{ \pm 1}$ and $Y_{2}^{ \pm 1}$ both change one moment quantum, they are essentially different. Notice that the non-zero matrix elements of $Y_{2}^{ \pm 1}$ have a sign difference but $Y_{1}^{ \pm 1}$ have no such term. If single quantum transition channels are in-phase, it is a dipole; if they are out-of-phase, it is a quadrupole. Similar analysis can be applied to other super bases. A diagrammatic interpretation of the dipoledipole and quadrupole-quadrupole RKKY exchange interactions is shown in Fig.5.

\section{METHOD OF COMPUTING COUPLING MATRIX}

Here we introduce an efficient method to calculate the coupling matrix of a multipolar exchange interaction using total energy electronic structure calculation, such as $\mathrm{LDA}$ or $\mathrm{LDA}+\mathrm{U}^{42}$. The discussion is based on a specific total moment $J$, therefore labeling by $J$ will be omitted.

\section{A. Energy Variation}

Consider a multipolar exchange interaction within the mean-field approximation, and the ground state energy
$E_{0}$ :

$$
\begin{aligned}
H & =\sum_{i j} \sum_{K Q} C_{K_{i} K_{j}}^{Q i Q_{j}} T_{Q_{i}}^{K_{i}} T_{Q_{j}}^{K_{j}} \\
& \simeq \sum_{i j} \sum_{K Q} C_{K_{i} K_{j}}^{Q i Q_{j}}\left\{\left\langle T_{Q_{i}}^{K_{i}}\right\rangle T_{Q_{j}}^{K_{j}}+T_{Q_{i}}^{K_{i}}\left\langle T_{Q_{j}}^{K_{j}}\right\rangle\right\} \\
E_{0} & =\langle H\rangle=2 \sum_{i j} \sum_{K Q} C_{K_{i} K_{j}}^{Q i Q_{j}}\left\langle T_{Q_{i}}^{K_{i}}\right\rangle\left\langle T_{Q_{j}}^{K_{j}}\right\rangle .
\end{aligned}
$$

The formula for the ground state energy is exactly the classical version of the multipolar exchange interaction. In this case, the multipolar moments are no longer quantized and can vary continuously. If we introduce variations for a particular pair of multipoles at different sites:

$$
\begin{aligned}
\left\langle T_{Q_{i}}^{K_{i}}\right\rangle & \rightarrow\left\langle T_{Q_{i}}^{K_{i}}\right\rangle+\delta\left\langle T_{Q_{i}}^{K_{i}}\right\rangle \\
\left\langle T_{Q_{j}}^{K_{j}}\right\rangle & \rightarrow\left\langle T_{Q_{j}}^{K_{j}}\right\rangle+\delta\left\langle T_{Q_{j}}^{K_{j}}\right\rangle
\end{aligned}
$$

plug them into the formula for $E_{0}$, we obtain:

$$
C_{K_{i} K_{j}}^{Q i Q_{j}}(i, j)=\frac{1}{2} \frac{\delta^{2} E_{K_{i} K_{j}}^{Q i Q_{j}}}{\delta\left\langle T_{K_{i}}^{Q_{i}}\right\rangle \delta\left\langle T_{K_{j}}^{Q_{j}}\right\rangle}
$$

and

$$
\begin{aligned}
\delta^{2} E_{K_{i} K_{j}}^{Q i Q_{j}} & =\left(\delta E_{K_{i} K_{j}}^{Q i Q_{j}}-\delta E_{K_{i}}^{Q i}-\delta E_{K_{j}}^{Q j}\right) \\
\delta E_{K_{i}}^{Q i} & =E_{K_{i}}^{Q i}-E_{0}
\end{aligned}
$$

where $E_{K_{i}}^{Q i}$ is the new energy associated with a variation $\delta\left\langle T_{Q_{i}}^{K_{i}}\right\rangle$ of $\left\langle T_{Q_{i}}^{K_{i}}\right\rangle$ multipole. Therefore, in order to calculate a multipolar exchange constant we need to obtain three energies: the energy cost of making a variation on site $i$, the energy cost of making a variation on site $j$ and the energy cost of making the same variations on both sites. If the multipoles $T_{Q_{i}}^{K_{i}}$ and $T_{Q_{j}}^{K_{j}}$ are not coupled, the energy cost of varying both will be simply the sum of two independent variations on each site. However, if they are coupled, varying both sites simultaneously will induce an extra exchange energy which is proportional to the exchange constant as shown in Fig.6. Therefore, if one can compute these energies using advanced electronic structure calculation, one is able to obtain the effective exchange interaction.

Another issue is how to perform a variation on the multipoles in a realistic calculation. To answer this question, we have to use the trace inner product theorem. Consider the local density matrices of each correlated site. The local density matrices can be expanded by a super basis defined on that site: $\rho_{i}=\sum_{K_{i}, Q_{i}} \alpha_{K_{i}}^{Q_{i}} Y_{K_{i}}^{Q_{i}}$. We intentionally choose a super basis where all the tensor operators are Hermitian, e.g., cubic harmonics, so we also have: $\left\langle T_{K_{i}}^{Q_{i}}\right\rangle=\operatorname{Tr}\left[\rho T_{K_{i}}^{Q_{i}}\right]=\operatorname{Tr}\left[\rho T_{K_{i}}^{\dagger Q_{i}}\right]=\alpha_{K_{i}}^{Q_{i}}$. It means $\left\langle T_{K_{i}}^{Q_{i}}\right\rangle \rightarrow\left\langle T_{K_{i}}^{Q_{i}}\right\rangle+\delta\left\langle T_{K_{i}}^{Q_{i}}\right\rangle$ is essentially $\alpha_{K_{i}}^{Q_{i}} \rightarrow \alpha_{K_{i}}^{Q_{i}}+\delta \alpha_{K_{i}}^{Q_{i}}$. Therefore, we can vary a multipole by changing its corresponding expansion coefficient. 


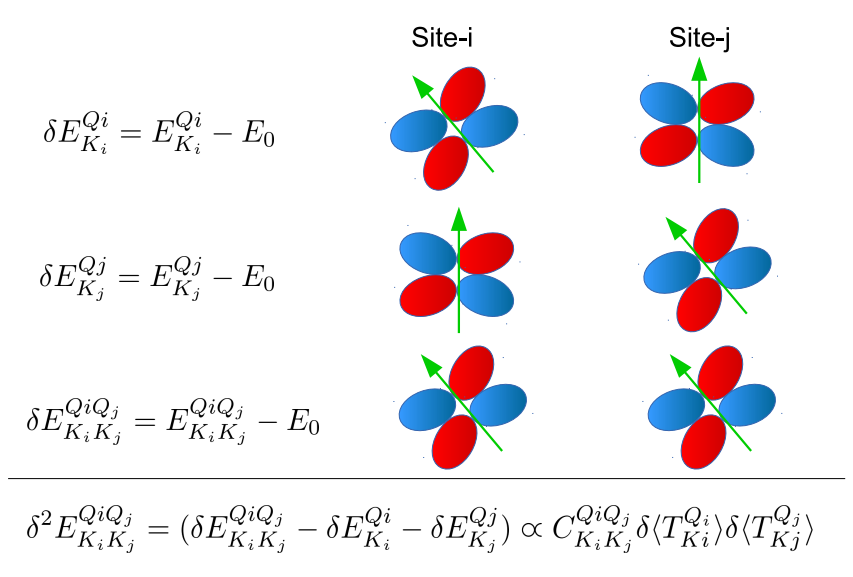

FIG. 6. (color) The meaning of each term in computing coupling constants. Here we use $T^{y z}$ to represent the multipoles.

\section{B. Pair-flip Technique}

When mapping the exchange model Hamiltonian to a series of total energy calculations using our method, it is necessary to make sure the total energies only contain the contributions from exchange interactions. This is not very straightforward. Recall that, in $S=1 / 2$ Heisenberg model, we relate the exchange constants to the new total energy of the meta-stable state with one or more spin moments flipped. Therefore when calculating the total energy, one should not perform any self-consistent calculation of the tensor flipped configuration else the system may evolve and go back to its ground state. To avoid the latter, we use the Anderson force theorem 45 and read the band energy differences only, i.e. the energies associated with occupied single--particle states. We also apply several constraints on the variations: 1) keep the total charge conserved; 2) keep the symmetry to avoid crystal field effects; 3) keep the magnitude of the multipolar moments fixed; 4) enforce the hermitianness of the density matrix. Combining these constraints, the only possible choice is to add a phase on the expansion coefficients $\alpha_{K_{i}}^{Q_{i}}$ of the density matrix $\rho_{i}=\sum_{K_{i} Q_{i}} \alpha_{K_{i}}^{Q_{i}} Y_{K_{i}}^{Q_{i}}$ and the simplest one is a minus sign: $\alpha_{K_{i}}^{Q_{i}} \rightarrow-\alpha_{K_{i}}^{Q_{i}}$. When this is done, $\delta \alpha_{K_{i}}^{Q_{i}}=-2 \alpha_{K_{i}}^{Q_{i}}$. This is similar to the way we calculate the exchange constants in conventional $S=1 / 2$ Heisenberg model, i.e. relating the exchange constants to the energy cost of flipping a spin moment (changing the sign of the $z$-axis spin projection).

However, the term "flip" has a different meaning in the language of multipolar exchange interactions from the case of $S=1 / 2$. In the conventional $S=1 / 2$ Heisenberg model, a "flip" means the flipping of the local axis of a spin moment. For most multipoles, such a flip is meaningless because it generates no change. Instead, the most general concept of a "flip" is to put a minus sign on their expansion coefficients or, equivalently, flip their phase ( a $\pi$ phase gives us $e^{i \pi}=-1$ ). In Fig.7, we show

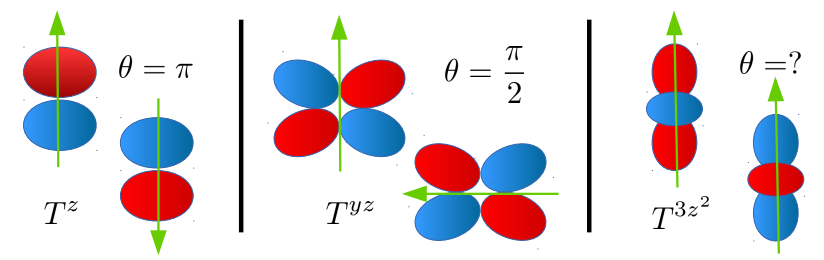

FIG. 7. (color) Phase flip of multipoles. A phase flip is different form the axis flip. For $T^{y z}$, it is a $\pi / 2$ rotation along the $x$ axis. For $T^{3 z^{2}}$, it does not correspond to any rotation. Only dipoles fit the concept of flipping their axes.

the pictures of a "phase flip" for cubic harmonics tensor operators via changing the sign on their corresponding functions. For dipoles, it is indeed equivalent to flipping its local axes. However, for quadrupoles, a phase flip of $T^{x y}, T^{y z}, T^{z x}$ and $T^{x^{2}-y^{2}}$ is actually a $\pi / 2$ rotation along different axes, and for $T^{3 z^{2}}$, it cannot be characterized as a rotation. Therefore, when we say "antiferromagnetic" $T^{y z}$ quadrupolar ordering, it actually means the $T^{y z}$ quadrupoles are ordered by a $\pi / 2$ rotation alternatively ${ }^{10}$ or, more precisely, by a $\pi$ phase change.

Now, we summarize how to calculate the exchange constants using the pair-flip technique: 1) Calculate the ground state of the system using advanced electronic structure calculation, such as LDA or LDA+U. 2) Generate an appropriate super basis that is consistent with the symmetry of the system. 3) Expand the local density matrices of the magnetic orbitals in this super basis. 4) Pick a pair of tensor components on different sites, flip their phases separately and simultaneously, recombine them into new local density matrices (make sure they are still Hermitian) and calculate their corresponding band energies (making sure not to do any self-consistent calculation on these meta-stable states). 6) Read the band energies and calculate the exchange coefficients.

\section{APPLICATION TO URANIUM DIOXIDE}

\section{A. Model Hamiltonian}

To test our method, we use Uranium Dioxide $\left(\mathrm{UO}_{2}\right)$ as a benchmark material due to the presence of dipolar and quadrupolar order in its ground state. $\mathrm{UO}_{2}$ has been one of the widely discussed actinide compounds due to its applications in nuclear energy industry. It is a Mott insulator with cubic structure and well-localized $5 f^{2}$ electrons (Uranium valence is $\mathrm{U}^{4+}$ by naive charge counting). Below $T_{N}=30.8 \mathrm{~K}$ it undergoes a first-order magnetic and structural phase transition where a non-collinear antiferromagnetic (AFM) phase with transverse 3-k magnetic ordering accompanied by the cooperative JahnTeller distortion occurs 11 . The two-electron ground state forms a $\Gamma_{5}$ triplet, holding pseudospin $J=1$ rotation 
symmetry 10

$$
\begin{aligned}
|+\rangle & =\sqrt{\frac{7}{8}}|+3\rangle-\sqrt{\frac{1}{8}}|-1\rangle ; \quad\left\langle+\left|J_{z}\right|+\right\rangle=\frac{5}{2} \\
|0\rangle & =\sqrt{\frac{1}{2}}|+2\rangle-\sqrt{\frac{1}{2}}|-2\rangle ; \quad\left\langle 0\left|J_{z}\right| 0\right\rangle=0, \\
|-\rangle & =-\sqrt{\frac{7}{8}}|-3\rangle+\sqrt{\frac{1}{8}}|+1\rangle ; \quad\left\langle-\left|J_{z}\right|-\right\rangle=-\frac{5}{2} .
\end{aligned}
$$

The numbers in the kets of the right-hand-side label the $m_{J}$ of the ${ }^{3} H_{4}$ configuration. It makes $U O_{2}$ a good choice to test our method, as it is a minimal challenge beyond $S=1 / 2$ Heisenberg model.

As discussed in the previous sections, the description of a spin-orbit coupled $J=1$ system requires the existence of dipolar and quadrupolar moments. It is commonly believed that there are two major mechanisms to induce exchange coupling in this system: 1) superexchange (SE), and 2) spin-lattice interaction (SL). The former contributes to both dipole and quadrupole and the latter contributes to quadrupole only due to the symmetry of structural lattice distortion. The dominance of $\mathrm{SE}$ or SL in affecting the quadrupole exchange remains a controversial issue $\mathrm{10}^{10}$. Since our method is based on a static electronic structure calculation, we do not explore dynamical effects in all their details. Therefore, separate calculations using the coupled frozen-phonon and frozen-magnon techniques were performed to extract the SL coupling constants.

Since $\mathrm{UO}_{2}$ is a cubic system, it is natural to take cubic harmonics as our super basis: $T^{s}$ for rank $0 ; T^{x}$, $T^{y}, T^{z}$ for rank 1 (dipole); $T^{x y}, T^{y z}, T^{z x}, T^{x^{2}-y^{2}}, T^{3 z^{2}}$ for rank 2 (quadrupole)1,10,12. The ground state local density matrix of an $\mathrm{U}$ ion can be expanded by them $\rho_{i}=\sum_{m} \alpha_{i}^{m} T_{i}^{m}$, where $i$ is site index, $m$ is the projection index for cubic harmonics, and $\alpha_{i}^{m}=\operatorname{Tr}\left[\rho_{i} T_{i}^{\dagger m}\right]$ is the expansion coefficient. Since the triplet degeneracy of $\Gamma_{5}$ is further split below $T_{N}$, we can approximate the ground state as $|G S\rangle=|-1\rangle$, the lowest energy state of an isolated $\mathrm{U}$-ion in the $3-\mathbf{k}$ magnetic phase. $3-\mathbf{k}$ ordering requires the four $U$ sublattice moments to point in inequivalent $(1,1,1)$ directions, which means the $|-1\rangle$ states are defined in different local coordinates for each $U$ sublattice $\underline{11}$. Thus, we need to make a rotation on each site to ensure everything is in a global coordinate system.

In the local coordinate system, the expansion of density matrices has the same tensor expansion coefficients:

$$
\rho_{i}=|-1\rangle\langle-1|=\sqrt{\frac{1}{3}} T_{i}^{s}-\sqrt{\frac{1}{2}} T_{i}^{z}+\sqrt{\frac{1}{6}} T_{i}^{3 z^{2}}
$$

When converting to global coordinate system, one has to apply a rotation matrix $D\left(\theta_{i}, \phi_{i}, \psi_{i}\right)^{\dagger} \rho_{i} D\left(\theta_{i}, \phi_{i}, \psi_{i}\right)$ using different Euler angles $\left(\theta_{i}, \phi_{i}, \psi_{i}\right)$ for each site. Then nonvanishing components of the ground state $3-\mathbf{k}$ quadrupolar order are $s, x, y, z, x y, y z$ and $z x$. Thus the model Hamiltonian of nearest-neighbor exchange interaction between magnetic $U$ atoms is assumed to be (in

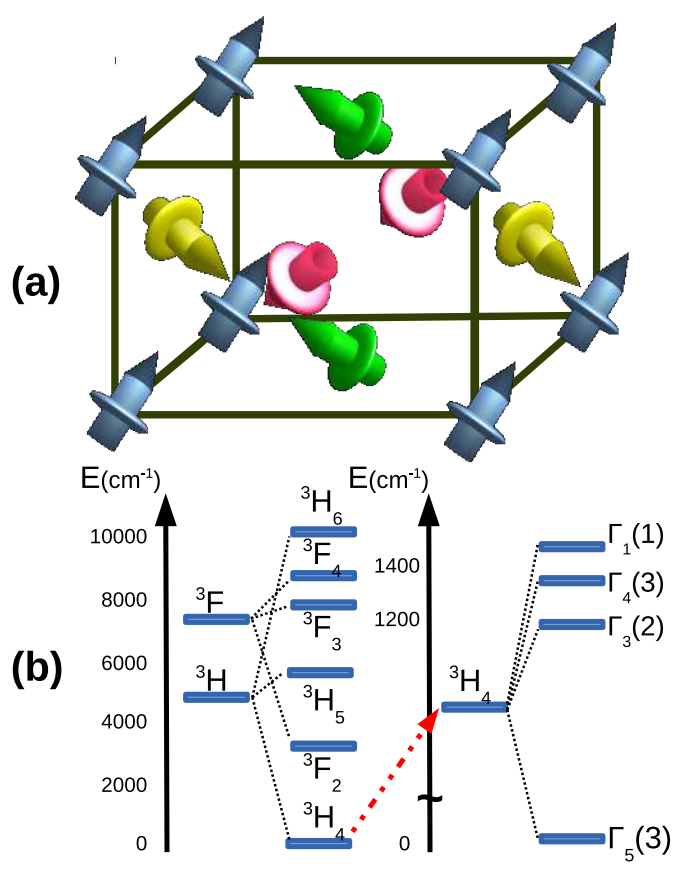

FIG. 8. (color) (a) Magnetic moments of dipoles (arrows) and quadrupoles (disk) in the 3-k structure. (b) The energy splitting of low lying states in $\mathrm{UO}_{2}{ }^{11}$. The ${ }^{3} \mathrm{H}$ and ${ }^{3} \mathrm{~F}$ states of free $U^{4+}$ ion are split into ${ }^{3} H_{4}$ multiplets and other excited states by spin-orbit coupling and further split into the $\Gamma_{5}$ triplet ground state by crystal fields. Their degeneracy are shown inside the parentheses

the global system):

$$
\begin{aligned}
h^{E X} & =h^{S E}+h^{S L} \\
& =\sum_{m i j} C_{i j}^{m m} T_{i}^{m} T_{j}^{m}+\sum_{n i j} K_{i j}^{n n} T_{i}^{n} T_{j}^{n} \\
& m \in x, y, z, x y, y z, z x \quad ; \quad n \in x y, y z, z x,
\end{aligned}
$$

where $(i, j)$ are the nearest-neighbor site indexes and $\left(C_{i j}^{m m}, K_{i j}^{n n}\right)$ are the exchange constants from SE and SL respectively. Couplings between tensor operators with different symmetry indexes are prohibited by cubic symmetry. This fact demonstrates the importance of choosing an appropriate super basis. The originally unknown $9 \times 9=81$ superexchange coupling constants now become only 6 .

\section{B. Superexchange Coupling}

Due to the 3-k symmetry, one can perform the pairflip technique on an arbitrary pair of uranium atoms in the four sublattices and all other exchange constants can be obtained by permuting their corresponding $x, y z$ coordinates. There are four equivalent bonds for a pair of uranium sites $(i, j)$, so to eliminate double counting one should also divide the obtained exchange energies by 4 as 
TABLE I. Comparison between our calculated exchange interaction parameters using the $\mathrm{LDA}+\mathrm{U}$ method with $U=4.0$ $\mathrm{eV}$ and $J=0.7 \mathrm{eV}$ and the existing experimental fits. $C_{0}^{d}, C_{0}^{q}$, $K_{0}^{q}$ are in units of meV, others are dimensionless. Because all the works use different models to simulate the SL part, there is no appropriate values for them (labeled by ${ }^{*}$ ). Ref ${ }^{13}$ obtained SL via a fully dynamic calculation. Note also that Ref. ${ }^{10}$ assumes the quadrupolar coupling only comes from SL with real space exchange constant of the $3-k$ symmetric form: $K_{i j}^{\Gamma}=K_{0} e^{i \mathbf{q}_{\Gamma}\left(\mathbf{R}_{i}-\mathbf{R}_{j}\right)}$. Ref ${ }^{11}$ only calculates SE part. Their parameters were obtained via the integrals of Coulomb interaction directly and have no simple anisotropy form.

\begin{tabular}{lcccccc}
\hline \hline \multicolumn{1}{c}{ Ref. } & $C_{0}^{d}$ & $\chi_{c}^{d}$ & $C_{0}^{q}$ & $\chi_{c}^{q}$ & $K_{0}^{q}$ & $\chi_{K}^{q}$ \\
\hline our work & 1.70 & 0.3 & -3.10 & 0.90 & 2.6 & 1.18 \\
$\underline{13}$ & 3.1 & 0.25 & 1.9 & 0.25 & $*$ & $*$ \\
$\underline{10}$ & 1.25 & 0.8 & 0 & 0 & 0.33 & $*$ \\
$\underline{11}$ & $\sim 1$ & $*$ & $\sim 0.1$ & $*$ & $\times$ & $\times$ \\
\hline \hline
\end{tabular}

well as account for any geometric or trigonometric factors due to the non-collinear order. Since $\left|\Gamma_{5}\right\rangle$ ground state is defined in the pseudospin $J=1$ space, we shall introduce the reduced density matrix (RDM) as a useful single-particle approximation to make it compatible with the single-particle based electronic structure calculation. However the self-consistent ground state of the $\mathrm{UO}_{2}$ may be close to but not equal to the RDMs of the prefect $\left|\Gamma_{5},-1\right\rangle$ state, so we keep all the calculated results unchanged but replace the local density matrices of the correlated orbitals by prefect $\left|\Gamma_{5},-1\right\rangle$ RDMs to make our system a well-defined $\left|\Gamma_{5}\right\rangle$ problem. We assume that the multipolar exchange Hamiltonian in the $J=5 / 2 \oplus 7 / 2$ single-particle space is built by replacing all tensor operators, density matrices, and mean values in $J=1$ space to their corresponding single-particle RDM: $\left\langle T_{i}^{m}\right\rangle \rightarrow\left\langle\mathscr{T}_{i}^{m}\right\rangle,\left\langle\rho_{i}\right\rangle \rightarrow\left\langle\mathscr{D}_{i}\right\rangle$. The single-particle exchange Hamiltonian shares the same exchange constants as the $J=1$ two-particle version. Two things to notice here are: 1) the RDM exhibits $J=\frac{5}{2} \oplus \frac{7}{2}$ symmetry instead of $J=1$ and this means the rotation from local coordinates to the global coordinates has to be made in $J=1$ space, else the pseudospin quasiparticle description will be violated; 2) the RDM replacement will rescale the length of an operator, i.e. $\operatorname{Tr}\left[\mathscr{T}^{\dagger}\right] \neq \operatorname{Tr}\left[T T^{\dagger}\right] . \quad$ Therefore, $\left\langle\mathscr{T}_{i}^{m}\right\rangle=\operatorname{Tr}\left[\mathscr{D} \mathscr{T}^{\dagger}\right]$ is different from $\left\langle T_{i}^{m}\right\rangle=\operatorname{Tr}\left[\rho T_{i}^{\dagger m}\right]$. So one has to be cautious when using Eq. (9).

In Fig. 9, we have plotted the total energies obtained from our $\mathrm{LDA}+\mathrm{U}$ calculation. The blue bars are the sum of flipping the multipolar moment at site $i$ and $j$ individually and the red bars are obtained by flipping both of them simultaneously. The exchange energy $E^{E X}=\delta^{2} E_{K_{i}, K_{j}}^{Q_{i}, Q_{j}}=\delta E_{K_{i}, K_{j}}^{Q_{i}, Q_{j}}-\delta E_{K_{i}}^{Q_{i}}-\delta E_{K_{j}}^{Q_{j}}$ is just the difference between the two bars. One may notice that the exchange energy of the quadrupoles is much smaller than the one of the dipoles. This is because the multipolar moments $\left\langle\mathscr{T}_{i}^{m}\right\rangle$ are about an order smaller than the

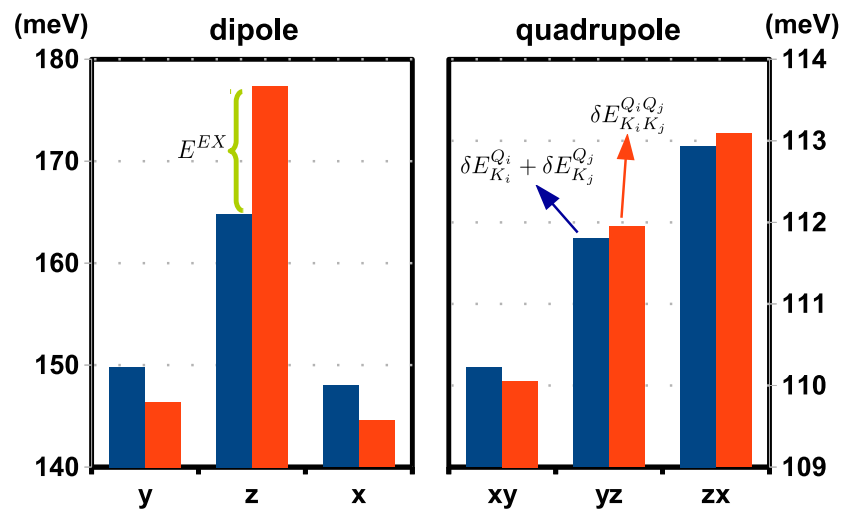

FIG. 9. (color) Calculted exchange energies using the LDA+U method with $\mathrm{U}=4.0 \mathrm{eV}$ and $\mathrm{J}=0.7 \mathrm{eV}$. The ground state energy (when nothing is flipped) is set to zero. Blue bars correspond to the sum over the energies of flipping a multipolar moment on site $i$ and $j$ individually. Red bars correspond to the energy of flipping the multipolar moments on site $i$ and $j$ simultaneously. Exchange energies are the differences between the red and blue bars (can be positive or negative).

dipoles. Once we include this factor, the exchange constants obtained using Eq.(9) are not necessarily small.

The coupling constants can be simplified by symmetry to the form:

$$
C_{i j}^{m n}=C^{m n}(\mathbf{R})=C_{0}^{d / q}\left[1-2\left(1-\chi_{c}^{d / q}\right) \tau_{m} \tau_{n}\right] \delta_{m n},
$$

where $d / q$ means dipole or quadrupole and $\tau=\mathbf{R} / R$ is the direction vector between $(i, j)$. These constants are shown in Table I, where the isotropic and anisotropic parts are described by $C_{0}^{d / q}$ and $\chi_{c}^{d / q}$ respectively 10 . With the comparison to other studies, the dipolar part is similar, but the quadrupolar part gives the opposite result to the past calculations obtained by best fit to experiment ${ }^{12,13}$. Not only the anisotropy effect is much smaller, but the sign is also different which means the quadrupoles tend to be ferromagnetic. It also means that the SL effects must be as important as SE and their combination makes the whole system antiferromagnetic.

\section{Spin-Lattice Coupling}

To explain the behavior of the quadrupolar part, we need to include the effect of dynamic contribution from SL. The coupling between spins and optical phonons can be written as:

$$
H_{S L}=\sum_{\mathbf{q} n j} V^{n}(\mathbf{q}, j) T^{n}(\mathbf{q}) u(\mathbf{q}, j),
$$

where $T^{n}(\mathbf{q})=\sum_{\mathbf{R}} T^{n}(\mathbf{R}) e^{i \mathbf{q} \cdot \mathbf{R}}, u(\mathbf{q}, j)=\left[a^{\dagger}(-\mathbf{q}, j)+\right.$ $a(\mathbf{q}, j)]$ and $a^{\dagger}(\mathbf{q}, j)$ is the creation operator of a phonon with wavevector $\mathbf{q}$ in mode $j$. Using the virtual phonon 
description, the SL exchange constant of $h^{S L}$ can be approximated as:

$$
K^{n n}(\mathbf{q}) \simeq \sum_{j} \frac{\left|V^{n}(\mathbf{q}, j)\right|^{2}}{h \omega(\mathbf{q}, j)}-\varepsilon_{0},
$$

where $\omega(\mathbf{q}, j)$ is the phonon frequency and $\varepsilon_{0}$ is the onsite exchange energy which should be subtracted 10 . The variables $u(\mathbf{q}, j)$ and $\omega(\mathbf{q}, j)$ have been calculated in one of our earlier works? and can be fitted to the entire Brillouin Zone using a simple rigid-ion model $\mathrm{l}^{48}, \underline{49}$. If we further assume that the quadrupoles only couple to $t_{2 g}^{a}$ and $t_{2 g}^{b}$ quadrupolar distortions of the $\mathrm{O}$-cage around each U-ion, the coupling constants are assumed to have the form:

$$
V^{n}(\mathbf{q}, j)=\gamma_{a} \psi_{a}^{n}(\mathbf{q}, j)+\gamma_{b} \psi_{b}^{n}(\mathbf{q}, j),
$$

where $\gamma_{a / b}$ are the parameters to be determined, $\psi_{a / b}^{n}(\mathbf{q}, j)$ are the inner product (projection) between the phonon distortion $u(\mathbf{q}, j)$ and $t_{2 g}^{a / b}$ distortion, and $u(\mathbf{q}, j)$ can be regarded as the distortion due to a phonon mode ${ }^{50}$. We estimate the parameters $\gamma_{a / b}$ by using a coupled frozen-phonon and frozen-magnon technique: 1 ) Make a $t_{2 g}^{a / b}$ distortion of the $\mathrm{O}$-cage around an U-ion; 2) Flip a particular tensor component of the single-ion RDM on a particular site; 3) Calculate the correlation energies: $\delta^{2} E_{a / b}^{m n}=\left[\delta E_{a / b}^{m n}-\delta E_{a / b}^{0 n}-\delta E^{m 0}\right]$, where the first superscript is the symmetry index of the quadrupole and the latter index is of $t_{2 g}^{a / b}$. So $\delta^{2} E_{a / b}^{m n}$ is the extra energy of making "flip+frozen phonon distortion" simultaneously compared to the energies of individual "flip" plus individual "frozen phonon distortion"; 4) Then the parameters are roughly: $\gamma_{a} \sim \delta^{2} E_{a}^{m n} / \sqrt{2}\left\langle T^{m}\right\rangle \psi_{a}^{n}$ and $\gamma_{b} \sim \delta^{2} E_{b}^{m n} /\left\langle T^{m}\right\rangle \psi_{b}^{n}$. There is a factor $\sqrt{2}$ in $\gamma_{a}$ because when we make the same displacement of each coordinate component, the length of the total displacement is $\sqrt{2}$ larger than $t_{2 g}^{b}$. By assuming the unit of phonon vibration about $0.014 \AA$ (as is the static JahnTeller distortion $\frac{11}{}$ ) and making a $t_{2 g}$ distortion to be $3 \%$ of the lattice constant, we have: $\gamma_{a}=34 \mathrm{meV}$ and $\gamma_{b}=48 \mathrm{meV}$. We can access nearest-neighbor constants by calculating $K^{n, n}(\mathbf{q}, j)$ at $\mathbf{q}=[0,0,0]$ and $\mathbf{q}=\frac{2 \pi}{a}[1,0,0]$, and by a subsequent fit to a cosine function with the on-site exchange energy assumed to be the average of the curve $\underline{10}$. We then have: $K_{i j}^{m n}=$ $K^{m n}(\mathbf{R})=K_{0}^{q}\left[1-2\left(1-\chi_{k}^{q}\right) \tau_{m} \tau_{n}\right] \delta_{m n}$ with $K_{0}^{q}=2.6$ $\mathrm{meV}$ and $\chi_{k}^{q}=1.18$.

\section{Magnetic Excitation Spectrum}

Combined with the superexchange contribution and using the Green function method with random phase approximation $\underline{10}$, we calculate the magnetic excitation spectrum of $\mathrm{UO}_{2}$ that is shown in Fig. 10. We find that the values and the characteristics of our results (a)
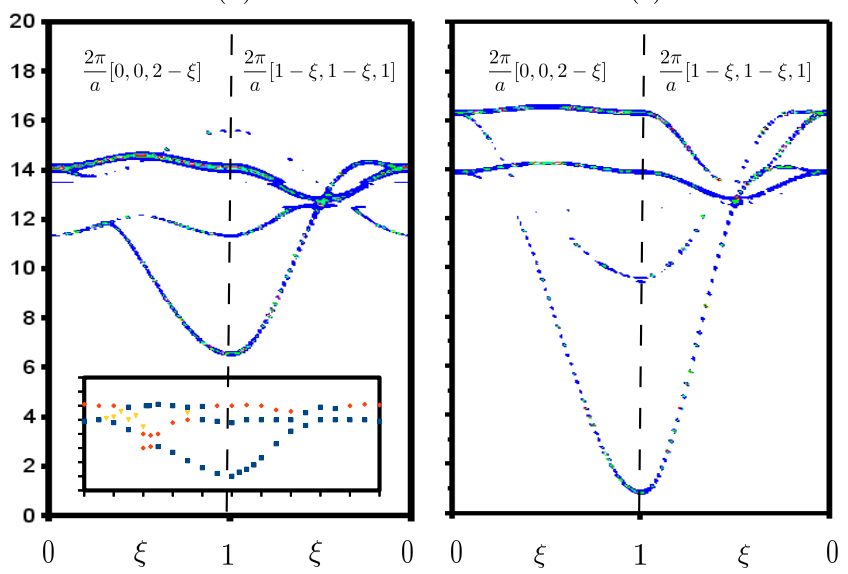

FIG. 10. (color) Magnetic excitation spectrum for $\mathrm{UO}_{2}$ along two symmetry directions calculated by scanning the colormap of the real part two-ion susceptibility of our model Hamiltonian ${ }^{10}$ with (a) parameters shown in Table I. (b) The same calculation made by requiring the overall quadrupole coupling to have 3-k symmetry: $K_{i j}^{\Gamma}=K_{0} e^{i \mathbf{q}_{\Gamma}\left(\mathbf{R}_{i}-\mathbf{R}_{j}\right)}$ with $K_{0}=0.5 \mathrm{meV}^{10}$ in which case the anisotropy gap is greatly reduced. Bottom inset: data from inelastic neutron scattering experiments plotted in the same $x-y$ scale ${ }^{46}$. Triangles (yellow) are measured in a direction set by a reciprocal lattice vector $^{47}$. Rhombus (orange) are weaker cross sections.

are basically in agreement with experiment. The major difference is the disappearance of anti-crossing at a few $\mathbf{q}$-points and much larger anisotropy (gap) at the $X$-point. The disappearance of the anti-crossing is reasonable because it comes from the coupling between magnon and phonon branches. As for the overestimated anisotropy at the $X$-point, it is believed to come from the oversimplified SL model in our calculation. We have plotted the spin/quadrupolar wave spectrum by enforcing the overall quadrupole coupling to have $3-k$ symmetry as Ref $\frac{13}{\underline{w}}$ with the parameter $K_{0}=0.5 \mathrm{meV}$ (which is almost the same value as our isotropic part) and it gives a much smaller gap which fits the experiment well (see Fig. 10). It demonstrates that a SL model which makes the whole quadrupolar coupling to have $3-k$ symmetry will be helpful in fitting the experiment but, in this case, the simplified form of our model will be also lost.

\section{CONCLUSION}

In conclusion, we have introduced the framework of multipolar operators and the benefits of using them as a language to describe the exchange interactions in spinorbit coupled systems. We have also developed a method to calculate the exchange constants via a density functional based total energy calculation. With its application to $\mathrm{UO}_{2}$, the superexchange tends to have ferromagnetic quadrupolar coupling rather than antiferromagnetic 
one which is very different from the past reports using best fits to experiments. It demonstrates that our method has the potential to explore magnetic spin-orbit coupled systems in more details. As for the spin-lattice interaction, we have performed a very similar calculation to estimate their couplings and the overall behavior is accounted for by the competition between the superexchange and spin-lattice counterparts. An accurate description of spin-lattice interactions and applications to hidden order systems would be beneficial for the future work.

\section{ACKNOWLEDGEMENT}

We are grateful to X. Wan and R. Dong for their helpful discussions. This work was supported by U.S. DOE Nuclear Energy University Program under Contract No. 00088708.
* spi@ucdavis.edu

† rnanguneri@ucdavis.edu

¥ savrasov@physics.ucdavis.edu

1 R. Caciuffo et. al., Rev. Mod. Phys. 81807 (2009)

2 Francesco Cricchio, Oscar Grns, and Lars Nordstrm, Phys. Rev. B. 81140403 (2010)

3 R. S. Gonnelli, D. Daghero, M. Tortello, G. A. Ummarino, V. A. Stepanov, J. S. Kim, and R. K. Kremer, Phys. Rev. B 79184526 (2009)

4 A. KISS and Y. KURAMOTO, J. Phys. Soc. Jpn. 742530 (2005)

5 H. SATO, T. SAKAKIBARA, T. TAYAMA, T. ONIMARU y, H. SUGAWARA, and H. SATO, J. Phys. Soc. Jpn. 76064701 (2007)

${ }^{6}$ H. C. Walker, K. A. McEwen, D. F. McMorrow, S. B. Wilkins, F. Wastin, E. Colineau, and D. Fort, Phys. Rev. Lett. 97137203 (2006)

7 T. Takimoto and P. Thalmeier, Phys. Rev. B 77045105 (2008)

8 Y. Kuramoto, H. Kusunose, and A. KISS, J. Phys. Soc. Jpn. 78072001 (2009)

9 P. Thalmeier, T. Takimoto, J. Chang and I. Eremin, J. Phys. Soc. Jpn. 7743 (2008)

10 P. Giannozzi and P Erdos, J. Mag. Mag Mater. 6775 (1987).

11 V. S. Mironov, L. F. Chibotaru and A. Ceulemans, Adv. Quan. Chem 44599 (2003)

12 S. Carretta et. al., Phys. Rev. Lett. 105167201 (2010)

13 R. Cauiuffo et. al., Phys. Rev. B 84104409 (2011)

14 P. Santini and G. Amoretti, Phys. Rev. Lett 852188 (2000)

15 P. Santini, S. Carretta, N. Magnani, G. Amoretti, and R. Caciuffo, Phys. Rev. Lett. 97207203 (2006)

${ }^{16}$ K. Kubo and T. Hotta, Phys. Rev. B 71140404 (2005)

17 D. Mannix, Y. Tanaka, D. Carbone, N. Bernhoeft, and S. Kunii , Phys. Rev. Lett. 95117206 (2005)

18 P. Chandra, P. Coleman, J. A. Mydosh V. Tripathi, Nature(London) 417831 (2002)

19 Francesco Cricchio, Fredrik Bultmark, Oscar Granas, and Lars Nordstrom, Phys. Rev. Lett. 103107202 (2009)

20 Hiroaki Ikeda, Michi-To Suzuki, Ryotaro Arita, Tetsuya Takimoto, Takasada Shibauchi and Yuji Matsuda, Nat. Phys. 8528 (2012)

21 A. Kiss and P. Fazekas, Phys. Rev. B 71054415 (2005)

22 J. G. Rau and H.-Y. Kee, Phys. Rev. B 85245112 (2012)
23 X. Wan, Q. Yin and S. Y. Savrasov, Phys. Rev. Lett. 97 266403 (2006)

24 J. R. Schrieffer and P. A. Wolff, Phys. Rev. 149491 (1966)

25 J. R. Schrieffer, J. Appl. Phys. 381143 (1967)

26 B. Coqblin and J. R. Schrieffer, Phys. Rev. 195847 (1969)

27 Stefan K. Kehrein and Andreas Mielke, Ann. Phys. 2521 (1996)

28 M. M. Salomaa, Phys. Rev. B 379312 (1988)

29 R. Siemann and B. R. Cooper, Phys. Rev. Lett. 441015 (1979)

30 D. Yang and B. R. Cooper, J. Appl. Phys. 522243 (1981)

31 P. Thayamblli, D. Yang and B. R Cooper, Phys. Rev. B 294049 (1984)

32 B. R. Cooper, J. Mag. Mag. Mater. 29230 (1982)

${ }^{33}$ N. Kioussis and B.R. Cooper, J. Mag. Mag. Mater. 54-57 701 (1986)

34 B. R. Cooper and R. Siemann, J. Appl. Phys. 501991 (1979)

35 Q. G. Sheng and B. R. Cooper, Phys. Rev. B 50965 (1994)

36 E. M Collins et. al., Phys. Rev B 621153 (2000)

37 J. M. Wills and B. R. Cooper Phys. Rev. B 424682 (1990)

38 J. M. Wills., B. R. Cooper and P. Thayamballi, J. Appl. Phys. 573185 (1985)

39 G.-J. Hu and B. R. Cooper, Phys. Rev. B 4812743 (1993)

40 P. W. Anderson, Phys. Rev. 12441 (1961)

41 Quantum Theory of Solids, 2ed. C. Kittel, Wiley (1987)

42 Electronic Structure Calculations for Solids and Molecles, J. Kohanoff, 1ed, Cambridge University Press (2006)

43 B. R. Cooper, A. Banerjea and P. Thayamballi, Physica 130B 117-121 (1985)

44 S.-T. Pi, R. Nanguneri, S. Savrasov, Phys. Rev. Lett. 112 077203 (2014)

45 A. R. Mackintosh and O. K. Andersen, Electrons at the Fermi Surface, edited by M. Springford (Cambridge University Press, England, 1990). p.149

46 R. Caciuffo et. al., Phys. Rev. B 5913892 (1999)

47 W. J. L Buyers and T. M. Holden, Handbook on Physics and Chemistry of the Actinides, Vol.2, edited by A. J. Freeman and G. H. Lander (Elsevier, Amsterdam, 1985), p239

48 E. W Kellermann, JSTOR 238-A 798(1940)

49 G. Dolling, R. A. Cowley, and A.D.B Woods, Can. J. Phys. 431397 (1965)

50 C.-Y. Huang and M. Inoue, J. Phys. Chem. Solids Pergamon Press 25889 (1964) 